\title{
Expression and Function of Ephrin Receptor B2 in Human Atherosclerosis: a Ligand Independent Guidance Cue
}

D. Vreeken ${ }^{1}$, C.S. Bruikman ${ }^{2}$, S.M.L. Cox ${ }^{1}$, H. Zhang ${ }^{1}$, A. Koudijs ${ }^{1}$, A.J. van Zonneveld ${ }^{1}$, G.K. Hovingh ${ }^{2}$, J.M. van Gils ${ }^{1}$ ${ }^{1}$ Department of Internal Medicine, Einthoven Laboratory for Vascular and Regenerative Medicine, LUMC, Leiden, The Netherlands, 'Department of Vascular Medicine, Amsterdam UMC, Amsterdam, The Netherlands

\section{Neuroimmune guidance cues in atherosclerosis}

Ephrin receptors (EPHs) and their ligands are related to a plethora of physiological and pathological processes including atherosclerosis. Preliminary data of our lab showed Ephrin Receptor B2 (EPHB2) to be the most abundantly expressed EPH receptor on monocytes, one of the culprit cell types in atherosclerosis. Combined with the observation that the EPHB2 receptor gene is located on a myocardial infarction locus, we hypothesized that monocytic EPHB2 plays a role in atherosclerosis, and set out to investigate the expression of EPHB2 in human atherosclerosis and its function in human monocytes.
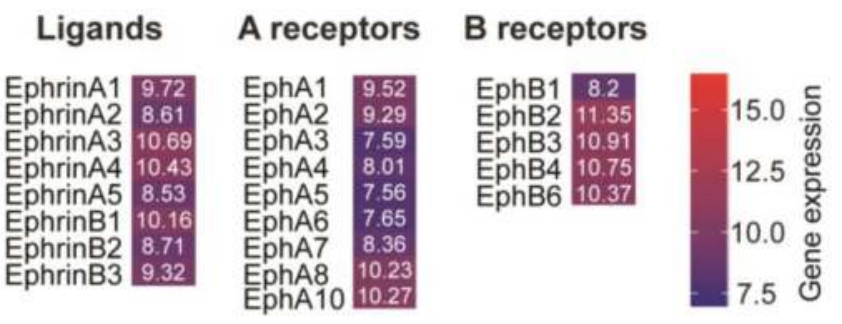

Increased expression of EPHB2 and EphrinB in progressive human atherosclerotic lesions
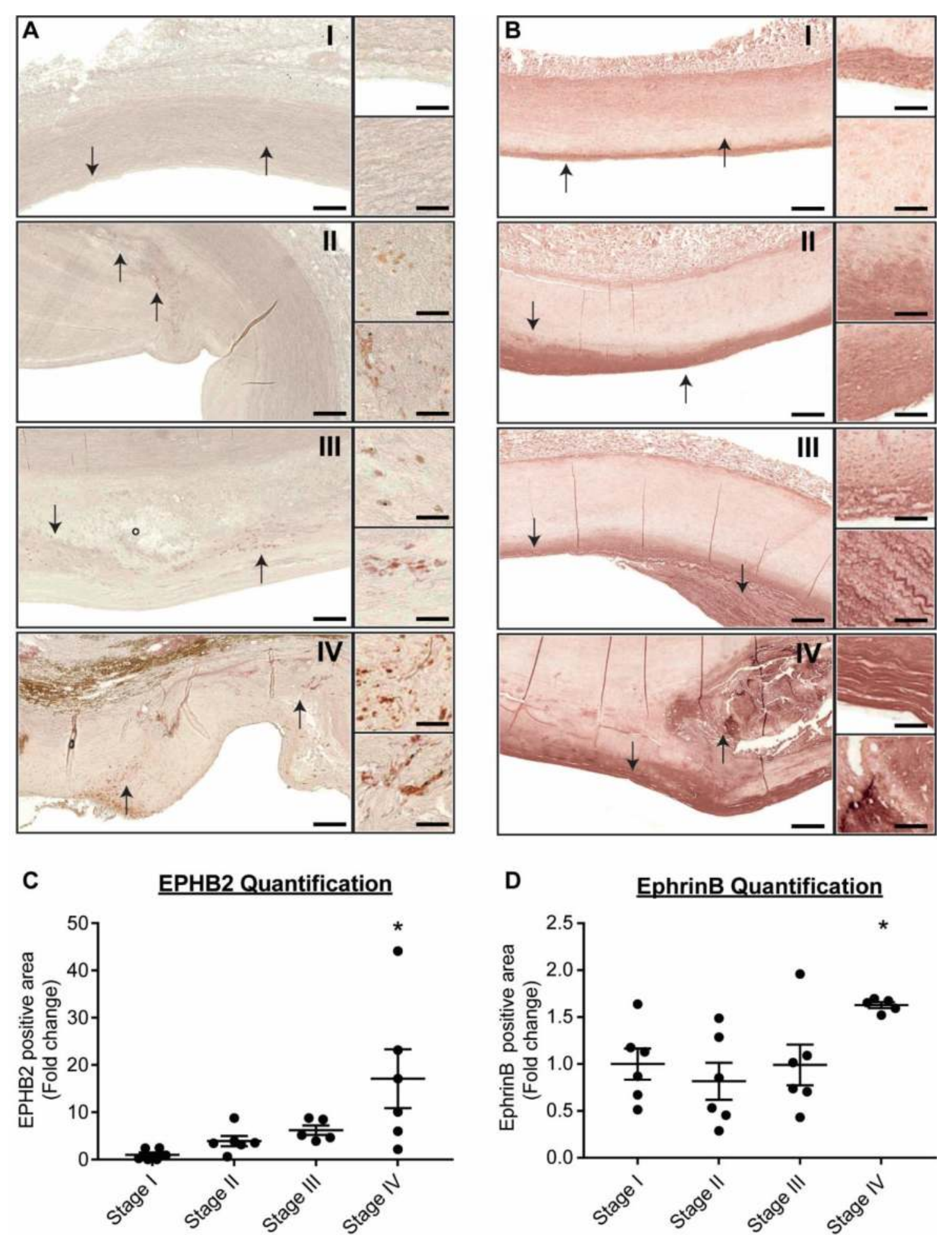

Immunohistochemical staining of human aortic tissue sections with varying stage of atherosclerosis for the receptor EPHB2 and its ligands EphrinB, showed an increase in expression of EPHB2 in the more progressive stages of disease $(A / C)$. Expression of the EphrinB ligands was high in all stages of plaque progression but significantly increased in the most severe stage of disease (B/D). Data is expressed as mean \pm SEM $* p<0.05$.
Decreased monocyte adhesion and migration upon knockdown of EPHB2

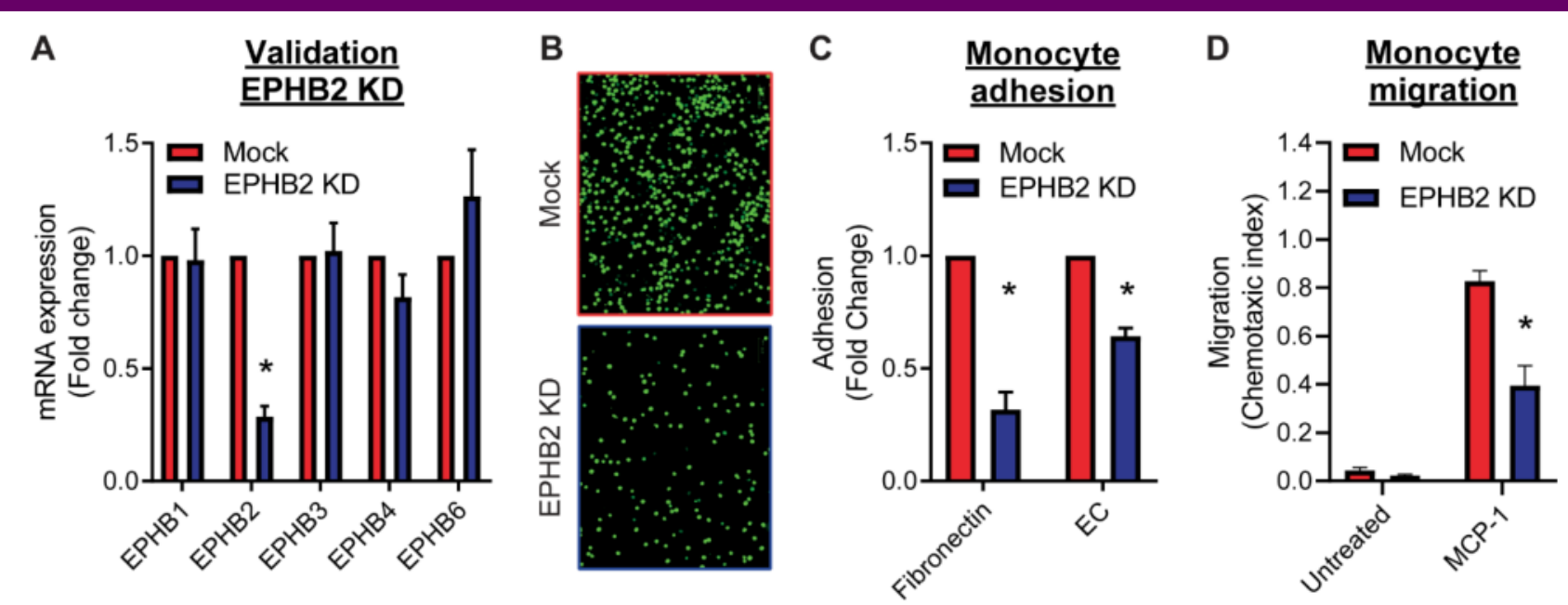

Specific lentiviral KD of EPHB2 in THP1 monocytes (A) resulted in a decreased capacity of monocytes to adhere to either fibronectin or a monolayer of HUVECS $(B, C)$. In addition transendothelial migration of monocytes towards MCP-1 is diminished in monocytes with a knockdown of EPHB2 (D). Data is expressed as mean \pm SEM $* p<0.05$.

\section{Effect of EPHB2 KD on monocyte adhesion and} migration is ligand independent
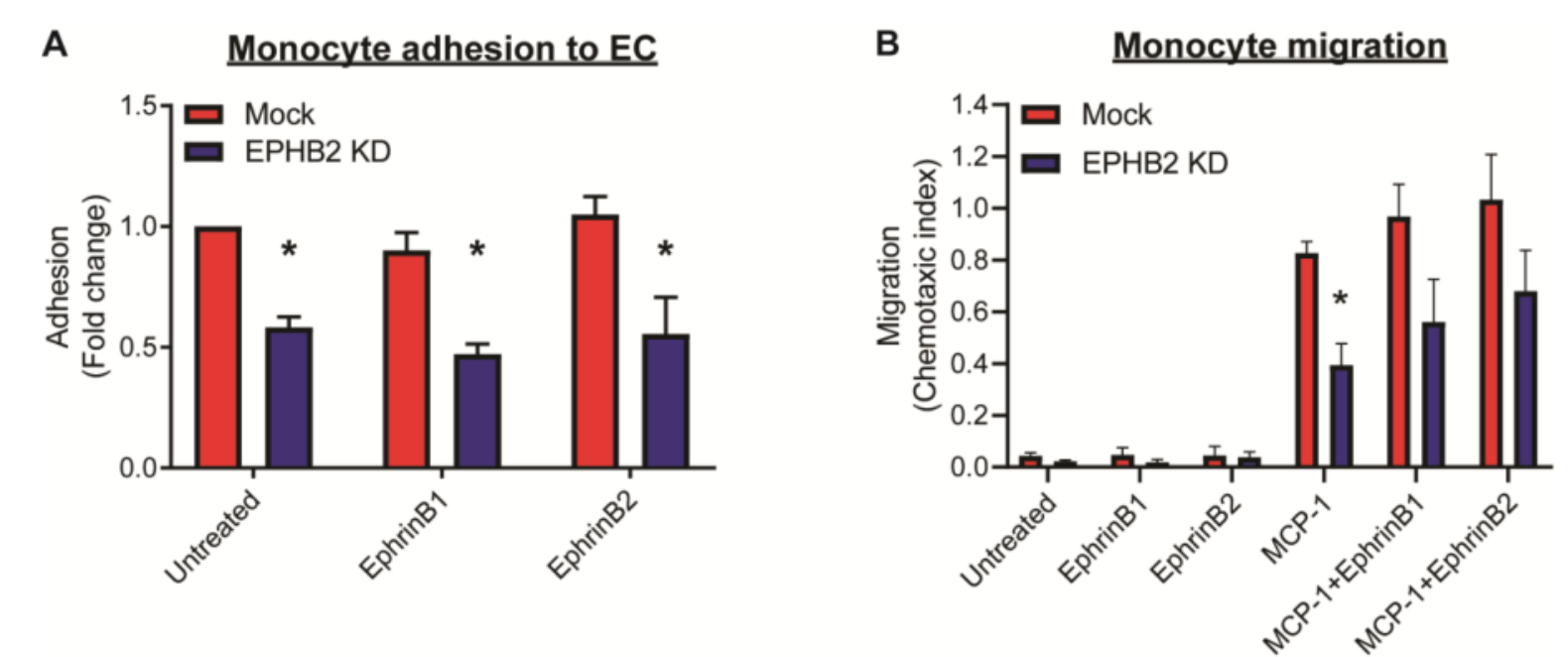

Stimulation of control or KD THP1 monocytes with recombinant EphrinB1 or EphrinB2 ligand did not alter the adherence of monocytes to endothelial cells (A), neither did migration towards MCP-1 change when either EphrinB1 or EphrinB2 was present (B). Data is expressed as mean $\pm S E M * p<0.05$.

EPHB2 KD alters cellular morphology and
phosphorylation patterns
Phalloidin staining of THP1 cells showed a rearranged
cytoskeleton with a decreased cell area in EPHB2 KD
cells compared to control cells (A, B). While mRNA
expression of adhesion modulators remains similar (C),
phosphorylation of FAK in EPHB2 KD cells is decreased
(D, E). Data is expressed as mean \pm SEM *p<0.05.

\section{Contact: d.vreeken@lumc.nl} EphrinB in atherosclerotic lesions is driven by atherosclerosis progression. In addition we have shown that EPHB2, independent of its ligands, facilitates monocyte adhesion and migration via regulation of the cytoskeleton of the cells and FAK phosphorylation, which in vivo might promote monocyte accumulation and thereby atherogenesis.

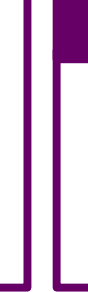

